\title{
Tigres azules de J. L. Borges. Lógica, verdad y mundos imposibles"
}

\author{
Fernán Rioseco P.**
}

\begin{abstract}
Resumen
El propósito de este artículo es mostrar el modo en que Borges, en su relato Tigres azules, desarrolla varias ideas interesantes en torno a la lógica, el lenguaje y las matemáticas. El punto de partida es el escepticismo borgeano sobre la capacidad de la lógica clásica para abordar el problema de la verdad y los mundos imposibles. Se defiende que objetos inconsistentes e, incluso, autocontradictorios no necesariamente dan lugar a mundos física o lógicamente imposibles. Se sugiere que las paradojas que Borges construye en el cuento no son paradojas de autorreferencia, sino variantes de la paradoja de Moore y de las paradojas de implicación material, por lo que para superarlas se requiere un enfoque no-clásico que neutralice $\mathrm{ECQ}$, esto es, un sistema de lógica paraconsistente.
\end{abstract}

Palabras clave: Borges, mundos imposibles, paradojas, lógica paraconsistente.

\begin{abstract}
Tigres azules of J. L. Borges. Logic, Truth and Impossible Worlds

The aim of this text is show how Borges, in his story Tigres azules, develops several interesting ideas about logic, language and mathematics. The starting point is Borgesian skepticism about the ability of classical logic to take the problem of truth and impossible worlds. It is defended that inconsistent and even self-contradictory objects do not necessarily give rise to physically or logically impossible worlds. It is suggested that the paradoxes that Borges constructs in the story
\end{abstract}

\footnotetext{
* Recibido: septiembre 2017. Aceptado: octubre 2017

*** Pontificia Universidad Católica de Valparaíso. Valparaíso, Chile. Email: flrioseco@gmail.com
} 
are not paradoxes of self-reference, but variants of Moore's paradox and paradoxes of material implication, so to overcome them requires a non-classical approach that neutralizes ECQ, this is, a paraconsistent logic system.

Keywords: Borges, impossible world, paradoxes, paraconsistent logic.

\section{Introducción}

El cuento Tigres azules de J. L. Borges (Borges, 1996a: 381-88) ha sido objeto de interpretaciones diversas. Algunas apuntan al problema de las relaciones entre orden y caos: ¿Cómo es que ha podido surgir el orden a partir del caos? ¿Qué relación existe entre orden y caos desde la perspectiva del pensamiento complejo? ¿De qué manera está presente en esta ficción la llamada teoría del caos? Otras lecturas ponen el acento en los aspectos semióticos del relato y en las paradojas lógicas y semánticas. También se ha identificado en el cuento vestigios de la influencia ejercida por Fritz Mauthner en la cuentística borgeana. Recientemente, se ha propuesto una mirada interdisciplinaria que considera elementos tomados de la estadística y de la teoría de probabilidades, con el fin de encontrar un patrón a una realidad numérica cambiante cuya variación parece gobernada por el caos (Kcenich y Luna-Escudero-Alié, 2015).

Borges mostró siempre un interés especial por la lógica, las paradojas y las contradicciones. Esta inclinación consta no sólo en Tigres azules, sino que es patente en varios de sus escritos filosóficos como Tlön, Uqbar, Orbis Tertius (Borges, 2011a: 723-36) y en el volumen de cuentos El libro de arena (Borges, 1996b: 11-73). En todos estos casos, junto a los problemas ontológicos y semánticos que conlleva la referencia a objetos imposibles o inexistentes (el volumen en octavo, los discos azules, las monedas de cobre de Tlön) Borges despliega la misma estrategia: superada la fascinación inicial por estos extraños y mágicos objetos, su portador ambiciona restablecer el orden more geométrico; restaurar la coherencia y armonía que supone inherentes a la realidad. Enfrentado a las paradojas y contradicciones que amenazan socavar los principios de la lógica, el portador (o el narrador en el caso de Tlön) se lanza a la búsqueda de un común denominador al que propendan estos objetos que se resisten al entendimiento. Acude, entonces, a sencillas operaciones lógicas y aritméticas, las que sin embargo se revelan infructuosas, lo mismo que a un cierto número de operaciones factuales que nada verifican en el mundo empírico. 
Tigres azules corresponde a la última etapa de Borges (1975-1985), en la que alcanza una de sus cumbres creativas en cuanto a la exposición de ciertos problemas lógicos, ontológicos y semánticos inherentes a la creación de ficciones literarias. Como el gran escritor que era, Borges soluciona con elegancia estas dificultades en un cierre que apela a elementos místicos y metafísicos. El desafío de este artículo es mostrar el modo en que estos problemas pueden ser abordados sin abandonar los dominios de la lógica.

Desde mi punto de vista, el eje central de Tigres azules es de naturaleza estrictamente lógica. Intentaré defender que Borges se muestra particularmente escéptico en cuanto a la capacidad de la lógica clásica para abordar el problema de los objetos imposibles y los mundos inconsistentes, así como las paradojas lógicas y semánticas (y su impacto en la noción de verdad proposicional). En la sección 1 expongo de manera sucinta la relación que Borges mantuvo con la lógica, especialmente la lógica matemática, con la idea de encontrar -si cabe- algún precursor entre esos lógicos. La sección 2 considera una breve explicación de las paradojas lógicas y semánticas. Se sugiere aquí que las paradojas de Tigres azules son variantes de la paradoja de Moore y de las paradojas de implicación material. En la sección 3 me ocupo de ciertos problemas ontológicos y semánticos que suscitan los objetos autocontradictorios en relación con los mundos imposibles. En la última sección describo las ideas esenciales del dialeteísmo y la lógica paraconsistente, e insinúo un bosquejo de solución de las paradojas de Tigres azules, sobre la base de la lógica de las paradojas (LP) de Graham Priest, incluyendo un cálculo paracompleto y una semántica paraconsistente de mundos imposibles.

\section{La lógica de Borges}

Sin perjuicio de precedentes que pueden hallarse en la obra de Leibniz, Bolzano y Boole, existe consenso en que el fundador de la lógica clásica fue el lógico alemán Gottlob Frege. En su Conceptografia (Begriffsschrift) el joven Frege es consciente del giro copernicano que para la lógica supone el lenguaje de fórmulas que acaba de inventar:

La mera invención de esta conceptografía, me parece, ha hecho prosperar a la lógica. Espero que los lógicos, si no se dejan intimidar por una primera impresión frente a lo extraño, no negarán su asentimiento a las innovaciones a que me vi impelido por una necesidad inherente al asunto mismo [...] En especial, creo que la sustitución de los conceptos de sujeto y predicado por los de argumento y función, se acreditará con el tiempo. Es fácil ver como la 
aprehensión de un contenido como función de un argumento surte el efecto de una aprehensión formadora de conceptos. Más aún, la demostración de la conexión entre los significados de las palabras sí, y, no, existe, algunos, todos, etc., merece atención" (Frege, 1972: 4; énfasis en el original).

El gran invento de Frege - los cuantificadores- y su perspectiva no psicologista, sentaron las bases de la lógica moderna, al grado que Begriffsschrift es considerada por muchos como el acta fundacional de la filosofía analítica. La influencia de Frege en pensadores del talante de Russell, Wittgenstein, Carnap y Quine es incuestionable, y el valor de su obra para lo que se ha venido en denominar la "tradición analítica" ha sido reivindicado por comentaristas de la talla de Michael Dummett y Hans Sluga.

Como se sabe, la obra de Borges abunda en referencias a la matemática, la lógica y la geometría. El autor argentino fue un ferviente lector y un aficionado competente en la exposición de problemas matemáticos como el infinito, los números transfinitos, la combinatoria y el cálculo probabilístico. En varios de sus escritos encontramos referencias a matemáticos y filósofos como Cantor, Peano, Russell, Whitehead, Stuart Mill, Brouwer, Mauthner y Meinong. Sin embargo, no hay en la vasta obra borgeana ninguna referencia a Frege. Me atrevo a sugerir que esto se debe a una omisión deliberada antes que a un olvido inexcusable, pues es claro que Borges debió conocer, a lo menos, las ideas fundamentales de Frege sobre la lógica y la filosofía del lenguaje. En efecto, si acaso Borges no lo leyó directamente, podemos suponer que accedió a su obra a través de Russell, cuya paradoja en la teoría de conjuntos manejaba bastante bien, igual que las distintas clases de infinitos matemáticos descubiertas por Cantor. Por otro lado, es posible que Borges haya tenido noticia de las ideas fregeanas debido a Fritz Mauthner, por cuanto las ideas de este último sobre la lógica y la teoría del lenguaje se hallan en las antípodas del pensamiento de Frege. Menos probable, aunque no puede descartarse, es que Borges haya accedido a la obra del lógico alemán siguiendo el derrotero de Wittgenstein y de los positivistas lógicos, pasando por Gödel, Carnap y Quine.

\subsection{Frege ¿precursor de Borges?}

En Kafka y sus precursores, Borges afirma que "cada escritor crea a sus precursores" (Borges, 1996c: 90). Oponiéndose a una visión historicista de la literatura y de la crítica literaria, el autor argentino construye un argumento similar al que Nietzsche utilizó sistemáticamente para rechazar las interpre- 
taciones historicistas de la historia. Según Borges, un precursor no es simplemente un autor que viene antes que otro y ejerce algún grado de influencia sobre las generaciones posteriores, sino algo más complejo y profundo. Un precursor es alguien que "modifica nuestra concepción del pasado, como ha de modificar el futuro" (Borges, 1996c: 90). Desde la perspectiva borgeana es posible y, en algunos casos, necesario, que un precursor no se encuentre antes, sino después de otro autor en la escala de temporalidad lineal.

Si se plantea el asunto de este modo, está claro que Frege no es un precursor de Borges. De las diversas alusiones a la lógica, la matemática y el lenguaje, no se infiere que Borges haya tenido la intención de crear a Frege como uno de sus precursores, ni mucho menos la de redimirlo. Es decidor que ni siquiera lo mencione, a diferencia de otros lógicos y matemáticos como Peano, Russell y Brouwer. En Tlön, Uqbar, Orbis Tertius leemos que en Tlön "la metafísica es una rama de la literatura fantástica" (Borges, 2011a: 729), sentencia que trasluce el pensamiento de Borges no sólo en lo relativo a su concepción de la filosofía, sino que de la realidad en su conjunto. Bajo el influjo de Mauthner, Borges es consciente de la naturaleza metafórica de todo lenguaje, como queda de manifiesto en El idioma analítico de John Wilkins (Borges, 1996d: 84-7). Refiriéndose a esta última ficción, Beatriz Sarlo afirma que "Borges subraya la cualidad arbitraria de todas las lenguas y provoca un escándalo lógico [...] El orden descompuesto, la lógica desencajada" (Sarlo, 2007).

Es precisamente esa lógica desencajada la que se muestra incapaz de hacer frente a las paradojas lógicas y semánticas. Como lo expresa Sarlo,

Borges admira las paradojas no porque sean incongruentes respecto de la experiencia sino por su demostración irónica de los límites de la lógica. Las paradojas no sólo trabajan con las inconsistencias o las contradicciones, sino que, obedeciendo a una dura coherencia formal, indican los límites de la lógica (sus escándalos) cuando se trata de aprehender la naturaleza lo real [...] Las paradojas son formas extraordinarias de la ficción (Sarlo, 2007: 101-2).

En un sentido similar, Bulacio identifica ciertos paralelismos entre la escritura borgeana (especialmente en su poética) y algunas ideas del primer Wittgenstein. Según ella "ambos autores tienen una posición semejante ante la metafísica, pero no la misma respuesta. Oscilan entre el rechazo de la metafísica como disciplina que pretende hablar de cosas extramundanas con el lenguaje del mundo y, al mismo tiempo, la fascinación por "otra realidad", de carácter metafísico que llama a silencio" (Bulacio, 2004: 113). El Wittgens- 
tein del Tractatus sabe que el lenguaje es demasiado limitado para acometer una empresa de esta envergadura. Por su parte, Borges no ignora que el absoluto está fuera del alcance de cualquier raciocinio, y por ello apela a ficciones pobladas de paradojas para no ofender a la razón y a la lógica.

Tengo para mí que Borges concebía la lógica (y la historia de la lógica) no como una demostración del intelecto humano, sino como un vano intento de superación de las paradojas y contradicciones. En palabras de Thiher,

Borges es un irónico sucesor de Cantor, Frege, Hilbert, Russell y Gödel, en el sentido de que celebra la destrucción de los sistemas que los lógicos matemáticos han tratado de apuntalar para superar las contradicciones que cada intento de pensamiento consistente parece hacer surgir [...] Este regocijo por la debilidad de la ciencia es cierto no sólo en el caso de contradicciones explícitas como en La Biblioteca de Babel, sino que también en las relaciones intertextuales entre las historias (Thiher, 2005: 243).

Más que irónico sucesor, puede decirse que Borges no es un precursor (y mucho menos un redentor) de ninguno de esos lógicos, por la sencilla razón de que era consciente de la fragilidad de la ciencia frente a las paradojas y antinomias. El análisis intertextual e hipertextual de los escritos borgeanos aporta, según Thiher, un dato consistente: para Borges las paradojas son inherentes al lenguaje y al pensamiento; y la lógica, en tanto lenguaje simbólico, no es inmune a ellas. Pero lo más grave es que la lógica no sólo carece de inmunidad frente a estos males semánticos y epistémicos, sino que es incapaz de hacerles frente satisfactoriamente. Allí donde hay pensamiento y lenguaje, emergen paradojas y contradicciones que constituyen un "escándalo lógico"1.

\section{Paradojas lógicas y semánticas}

En términos generales, una paradoja puede ser definida como una contradicción que resulta de una deducción correcta a partir de premisas congruentes. Dicho de manera tosca, una paradoja lógica es aquella que se origina al

\footnotetext{
${ }^{1}$ Algunos comentaristas defienden que en los escritos filosóficos de Borges no hay paradojas, sino rizomas. Borges no estaría interesado en las paradojas en tanto contradicciones dentro de un binarismo lógico, sino más bien en otros problemas como la infinita divisibilidad de los puntos, el desdoblamiento de las series infinitas y aleatorias, la abolición de la causalidad. La estructura de la paradoja borgeana no sería, entonces, la de una paradoja auténtica, sino la de una paradoja aparente. Su escritura es rizomática porque no se trata de contradicciones lógicas, sino de trazas nómadas, de una proliferación de significantes y significados (De Toro 1999: 174-5). A mi parecer, el argumento confunde el nivel hermenéutico con el nivel lógico. En la dimensión interpretativa podría sostenerse que la estructura de los cuentos borgeanos es rizomática, pero desde el punto de vista lógico las paradojas son genuinas. Vid. la sección 2.
} 
interior de un sistema de lógica formal. Según Russell las paradojas lógicas apuntan directamente a la consistencia de un sistema lógico, y se refieren a nociones lógicas o matemáticas tales como cardinal, ordinal, clases, relaciones, funciones, etc. Una paradoja de este tipo es la célebre paradoja de Russell en la teoría de conjuntos, de sobra conocida como para ser reproducida aquí. Otras paradojas lógicas son las de Burali-Forti (1897) y Cantor (1903).

De acuerdo con Pradilla, una paradoja semántica "es relativa a diversas nociones semánticas que relacionan el lenguaje con el mundo de los hechos reales, es decir, la noción de verdad verificacional de un enunciado o la noción de satisfacción de una fórmula, por una o varias entidades" (Pradilla, 2015: 92). Algunos ejemplos de paradojas semánticas son las de Rusell-Kaplan, Richard, Forrest-Armstrong, Berry, la del Mentiroso (atribuida a Epiménides), entre otras. Estas paradojas son típicamente autorreferenciales, en el sentido que se originan debido al uso contingente de expresiones del lenguaje natural o de términos lógicos que refieren recíprocamente al interior del propio sistema.

Aún cuando suelen emplearse como sinónimos salva veritate, en estricto rigor la paradoja es conceptualmente diversa de otra noción próxima como la contradicción (llamada, a veces, antinomia). Una contradicción "es una proposición acerca de la cual, puesta la hipótesis de su verdad, se deduce su falsedad y, puesta la hipótesis de su falsedad, el entendimiento descansa en ella y la confirma" (Díaz Estévez, 1972: 61).

\subsection{Las paradojas de Tigres azules}

En contraste con otros relatos borgeanos donde las paradojas se presentan de manera explícita, en Tigres Azules Borges recurre a una estrategia mucho más sutil, proporcionando una serie de pistas que parecen triviales, pero que en una lectura más reposada y en la medida que el cuento progresa hasta alcanzar su máxima tensión, denuncia las aporías de la razón, mostrándola estéril e incapaz de lidiar con las contradicciones del mundo real.

La estructura lógica de Tigres azules se divide en dos momentos nítidamente diferenciados:

a) El primero, que puede identificarse con la lógica clásica, comprende desde el inicio del relato hasta que Craigie ha aprehendido las piedras azules, ante el estupor y espanto de Bhagwan Dass, el miembro más anciano de la aldea. b) A partir de ese momento y hasta el final, la narración abandona el camino seguro de la lógica clásica, para ingresar 
de lleno en el mundo de los objetos imposibles, las paradojas y contradicciones, las aporías y antinomias de la razón. En este contexto, resulta tentador inferir que el carácter paradojal del cuento reside precisamente en esta tajante separación: la primera parte afirmaría el orden more geométrico, racional por antonomasia; mientras que la segunda estaría dominada por el caos y la irracionalidad. Estimo, sin embargo, que esta interpretación es errada: a mi entender, lo singular de Tigres azules consiste en que las paradojas que Borges construye no son autorreferenciales, sino variantes de la paradoja de Moore y de las paradojas de implicación material. No se trata, entonces, que el cuento tenga una "estructura paradójica", sino que su naturaleza paradojal deriva de que, en la segunda parte, se articulan paradojas lógicas y semánticas para cuya resolución la lógica clásica se revela ineficaz, siendo necesario acudir a lógicas no-clásicas. Lo esencial, en mi lectura de Tigres azules, es que nunca abandonamos los dominios de la lógica.

En abono de la tesis que aquí propongo, Borges entrega una serie de pistas que justifican la lectura en el sentido de que existiría un tránsito desde la lógica clásica hacia lógicas no-clásicas:

(i) La primera es el diseño del personaje principal, Alexander Craigie, un profesor escocés de lógica que se desempeña en la Universidad de Lahore, y que consagra sus domingos a un seminario sobre la obra de Spinoza. Hasta donde tengo conocimiento, en las diversas interpretaciones de Tigres azules no se ha reparado en el detalle de que Craigie es un profesor de lógica occidental y «oriental» en una Universidad de Pakistán (cerca de la frontera con India) y que, además, consagra parte de su tiempo a la obra de Spinoza. La explícita referencia de Borges a la lógica oriental no es casual, ni un dato anecdótico atribuible a su etapa "orientalista", sino que constituye la esencia del problema filosófico esbozado en el cuento: la insuficiencia de la lógica clásica para confrontar las paradojas y contradicciones y, por ende, la necesidad de acudir a lógicas no-clásicas. La misma importancia tiene la formulación de las siguientes preguntas: ¿Cuál es la lógica occidental que estudia y enseña Craigie? ¿Podrá tratarse de un sistema de lógica paraconsistente? ¿Qué relevancia tiene la obra de Spinoza en la concepción del mundo de Alexander Craigie? ¿Cuál Spinoza le interesa a Borges? ¿El Spinoza ético o el Spinoza político? ¿O ambos?

b) La segunda pista surge en la medianía del relato, cuando Craigie ya ha poseído las piedras azules. Borges recurre nuevamente a Spinoza: 
Para no pensar en los discos, para poblar de algún modo el tiempo, repetí con lenta precisión, en voz alta, las ocho definiciones y los siete axiomas de la Ética. No sé si me auxiliaron (Borges, 1996a: 384).

En la época en que Spinoza publicó su Ethica more geometrico demonstrata se pensaba - bajo el influjo del racionalismo cartesiano- que la geometría era el modelo de la ciencia perfecta y acabada, idea que Kant mantuvo en su Crítica de la Razón Pura y que Frege, en lo esencial, también conservó. Como apunta Stepanians, "en la perspectiva de Frege, la condición no sistemática de la aritmética se encontraba en claro contraste con los comparativamente más claros contornos de la geometría euclidiana, la cual se aproxima bastante al ideal de ser una ciencia probada y exacta" (Stepanians, 2007: 12).

Hasta aquí Borges se muestra respetuoso de la tradición filosófica, especialmente de la concepción racionalista defendida por Spinoza y Frege, con la salvedad que en el caso de Spinoza la geometrización tenía un propósito metafísico, mientras que para Frege se trataba de extender el método axiomático de la geometría a la aritmética para edificar esta última sobre las bases sólidas de la lógica.

c) La tercera pista la encontramos en el siguiente pasaje:

Si me dijeran que hay unicornios en la luna yo aprobaría o rechazaría ese informe o suspendería mi juicio, pero podría imaginarlos. En cambio, si me dijeran que en la luna seis o siete unicornios pueden ser tres, yo afirmaría de antemano que el hecho era imposible. Quien ha entendido que tres y uno son cuatro no hace la prueba con monedas, con dados, con piezas de ajedrez o con lápices. Lo entiende y basta. No puede concebir otra cifra. Hay matemáticos que afirman que tres y uno es una tautología de cuatro, una manera diferente de decir cuatro (Borges, 1996a: 385).

Estamos ya en la segunda parte del cuento. Craigie se resiste a abandonar el edificio de la lógica clásica, pues lo invade el temor de caer en la locura o, peor aún, tener que aceptar "la prueba de que en el universo cabe el desorden. Si tres y uno pueden ser dos o pueden ser catorce, la razón es una locura" (Borges, 1996a: 386). Borges se refiere a la imposibilidad de las piedras azules qua objetos, aceptando -incluso- que la existencia de unicornios en la luna sería posible (quizás a la manera de Meinong), pero en ningún caso la existencia de piedras que contradicen las más elementales reglas de la aritmética y hacen imposible el cálculo. Para Borges las piedras azules son un objeto imposible, esencialmente autocontradictorio, como el cuadrado-redondo o el 
Goofang incluido en su Libro de los seres imaginarios ${ }^{2}$. Y, en tanto objetos imposibles, engendrarían necesariamente un mundo imposible. Desde el punto de vista lógico, implica que todos los enunciados relativos a la aritmética deforme de las piedras azules son falsos en todos los mundos posibles. Dicho de otro modo, no hay un mundo posible en el que sea verdadero el condicional "Si tres y uno pueden ser dos o pueden ser catorce, entonces la razón es una locura", lo que constituye una manera oblicua de decir que el mundo de las piedras azules es un mundo imposible. Nótese que este tipo de inferencias son características de la lógica modal. Sin embargo, como espero mostrar a continuación, debido a la naturaleza esencialmente autocontradictoria de las piedras azules qua objetos, la lógica modal es insuficiente para superar las contradicciones, básicamente porque las paradojas de Tigres azules son variantes de la paradoja de Moore y de algunas paradojas de implicación material, para cuya solución es necesario acudir a lógicas no-clásicas.

\subsection{La paradoja de Moore}

Esta paradoja debe su nombre a G. E. Moore. Wittgenstein, quizá sin exagerar, afirmaba que era la contribución más importante de Moore a la lógica. En su forma simple la paradoja se presenta en la aserción de la oración $« p$ pero yo no creo que $p »$. La aparente absurdidad e irracionalidad de la paradoja se evidencia al traducir su forma lógica al lenguaje natural: «Está lloviendo, pero no creo que está lloviendo». Lo interesante es que ambas partes de la conjunción pueden ser verdaderas al mismo tiempo, sin que exista necesariamente una contradicción desde el punto de vista lógico. Para ser más precisos, la aserción no es formalmente autocontradictoria ${ }^{3}$. Por razones de espacio no puedo extenderme aquí en la paradoja, pero su importancia tiene que ver con las condiciones del discurso (verdad, sinceridad, insinceridad, racionalidad, etc.) y con las relaciones entre conocimiento y creencia. En el caso de Hintikka, por ejemplo, su estudio de las paradojas lo condujo a la distinción entre lógica epistémica y lógica doxástica, que esencialmente se apoya en la dicotomía saber-creencia (Hintikka, 1962).

La paradoja de Moore básicamente surge cuando un sujeto realiza una aserción en primera persona. En el caso de Tigres azules, la narración tiene precisamente esa particularidad. Hay un yo narrador que nos revela su obse-

\footnotetext{
${ }^{2}$ A diferencia del Goofus Bird, que es un ave extravagante, pero posible, el Goofang "nada para atrás para que no se le meta el agua en los ojos y es del tamaño exacto del pez rueda, pero mucho más grande". Esta descripción refiere propiedades autocontradictorias por lo que se trataría de un objeto imposible.

${ }^{3}$ En sentido contrario véase Shoemaker, 1995.
} 
sión por los tigres azules y su búsqueda insensata en "una aldea muy distante del Ganges (...) de cuyo nombre - por razones que luego aclararé- no quiero acordarme" (Borges, 1996a: 381-2). En la segunda parte, el monólogo de Craigie es fácilmente reconducible a oraciones mooreanas del tipo «Las piedras azules se multiplican, pero yo no creo que se multiplican»o «Las piedras se dividen, pero yo no creo que se dividen» y así, en general, con las cuatro operaciones de la aritmética. Otro tanto puede decirse de sus propiedades físicas: «Las piedras engendran, pero yo no creo que engendran». Desde el punto de vista de las condiciones del discurso (en este caso ficcional), la resistencia de Craigie a aceptar el errático comportamiento de las piedras parece sincera y auténtica. Su creencia es, además, consciente. No hay atisbos de locura en el personaje, fuera de su obsesión por los tigres azules. Por el contrario, su racionalidad estaría garantizada al ser profesor de lógica occidental y oriental en la Universidad de Lahore ("La Oxford del Oriente"). El discurso de Craigie es también consistente, pues enfrentado al "caos inextricable y a los indómitos discos", elige mantener la cordura y, en un salto de fe, acabar con el intolerable tormento pidiéndole a "Dios y Alá - dos nombres de un solo Ser inconcebible-" que lo libere de su carga.

En Tigres azules las oraciones mooreanas tienen una interesante variación asociada a la variable temporal. El propio Moore reconoció que las aserciones en tiempo pasado no eran necesariamente absurdas («Estaba lloviendo, pero yo no creía que estaba lloviendo»). En este sentido, las aserciones de Craigie se refieren a un cierto momento en la escala temporal, esto es, a un instante $t$ en que las piedras se dividen y multiplican. La forma en que Borges construye el relato da a entender que corresponde a una reconstrucción en primera persona de hechos pretéritos, lo que adquiere importancia a propósito de la doctrina dialeteísta que expongo más adelante.

\subsection{Las paradojas de implicación material}

Las características fundamentales de la lógica proposicional son: (i) la bivalencia y (ii) su funcionalidad de verdad. Es decir, esta lógica define el conjunto de inferencias cuya validez es función de la verdad (bivalente) de los enunciados. Así, la definición clásica de validez del condicional es: un argumento es válido si siempre que las premisas son verdaderas, entonces la conclusión no puede ser falsa. Dicho de manera trivial, no puede ocurrir que las premisas sean verdaderas y la conclusión falsa. 
Sin embargo, en dos casos extremos esta noción de validez del condicional produce resultados fuertemente contraintuitivos:

$$
\begin{aligned}
& \text { 1) } \mathrm{A} \Lambda \neg A \vdash B \\
& \text { 2) } A \vdash B v \neg B
\end{aligned}
$$

Estos casos dan origen a las llamadas paradojas de la implicación material $^{4}$, pues se trata de argumentos condicionales cuya validez y verdad semántica, la lógica clásica se ve compelida a aceptar, pese a que serían razonamientos contraintuitivos si se expresaran en lenguaje natural; a saber: 1) Si la luna es de queso, Francia está en Europa; 2) Si gano la partida de ajedrez, Trump es o no es Presidente de los Estados Unidos.

En Tigres azules las paradojas de implicación material se manifiestan a través de enunciados condicionales explícitos e implícitos. Una proposición del primer tipo es la siguiente:

1. Si tres y uno pueden ser dos o pueden ser catorce, la razón es una locura (Borges, 1996a: 386).

Otras veces el condicional material es implícito:

2. Si las cuatro operaciones de sumar, restar, multiplicar o dividir son imposibles, la aritmética es imposible.

En el caso de (1) tanto el antecedente como el consecuente son falsos (pues son autocontradictorios), mientras que en (2) el antecedentes es falso, pero el consecuente es verdadero.

En la lógica modal de C. Lewis (1) implicaría que en todo mundo posible donde tres y uno son dos, la razón es una locura, en tanto que (2) implica que en todo mundo posible donde las cuatro operaciones de sumar, restar, multiplicar o dividir son imposibles, la aritmética es imposible. Sin embargo, la dificultad lógica es que el antecedente «tres y uno son dos» y el consecuente «la razón es una locura», son falsos en todos los mundos posibles.

\footnotetext{
${ }^{4}$ La denominación «implicación material» es desafortunada, pues da a entender que el problema es de causalidad física o material, en circunstancias que es un problema de estricta consecuencia lógica. Por ello, se prefieren otras denominaciones, como "condicional material" que tiene la virtud de preservar esta última idea.
} 


\section{Mundos y objetos imposibles}

En Tigres azules las piedras son autocontradictorias en, al menos, dos sentidos: (i) Desde el punto de vista físico, porque "engendran" otras piedras que surgen de la nada, sin explicación racional ni causa aparente; (ii) Desde el punto de vista lógico, porque introducen una Aritmética deforme, donde las operaciones elementales de suma, resta, multiplicación y división son imposibles $^{5}$. Lo anterior conduce por fuerza a dos preguntas relacionadas: ¿Las piedras azules son objetos imposibles? Y, si lo son, ¿dan lugar necesariamente a mundos imposibles? La respuesta a estas preguntas no es para nada sencilla, pues no sólo implica considerar los sistemas de lógica modal, sino también el problema de la verdad en la ficción. Por razones de espacio, aquí apenas puedo esbozar algunas ideas básicas:

a) En primer lugar, debemos subrayar que en el caso de los operadores modales y las modalidades aléticas «posible», «necesario», «imposible» $\mathrm{y}$ «contingente», la mayoría de los filósofos no está pensando la imposibilidad en el sentido de la ciencia ficción (esto es, como mundos alternativos al nuestro), sino en una imposibilidad lógica. Si uno adhiere al esencialismo modal de D. Lewis, debe admitir que cualquier mundo posible existe de igual manera que nuestro mundo real, pues en este enfoque los mundos posibles son entidades concretas (Lewis, 1986: 2). Sin embargo, esta idea parece contraintuitiva, ya que supone aceptar la existencia de un mundo en el que Alicia va al país de las maravillas; otro en el que los dinosaurios no se extinguieron, etc. Por esta razón, algunos lógicos como Adams y Plantinga defienden un realismo modal moderado, en el que los mundos posibles son entidades abstractas que referirían a proposiciones y estados de cosas o a conjuntos de proposiciones y estados de cosas.

b) En cuanto a la noción de objeto imposible ${ }^{6}$ no existe consenso entre los filósofos, pues la respuesta dependerá del compromiso ontológico que subyace a la adopción de un determinado sistema de lógica formal. Por ejemplo, un defensor del formalismo matemático probablemente diría que un objeto es imposible siempre que sea contradictorio o autocontradictorio, como un triángulo de seis lados, el cuadrado-redondo o el Goofang incluido en el bestiario de seres imaginarios del propio Bor-

\footnotetext{
${ }^{5}$ Sin embargo, hay aquí un detalle en el que Borges no profundiza: él afirma que en estas condiciones el "cálculo es imposible", pero Craigie aún puede contar las piedras, por lo que incluso en el aterrador escenario de las "piedras que engendran" sigue existiendo una Aritmética no sólo posible, sino que real.

${ }^{6}$ Sobre el concepto de objeto imposible en Husserl y Meinong, cfr. Millán-Puelles, 1990.
} 
ges. En este enfoque ontológico, «imposible» equivale a inexistente. A la misma conclusión (inexistencia) llega el lógico clásico, aunque por razones diferentes a las del formalista. Sin embargo, es casi seguro que un defensor del realismo meinongniano, del posibilismo o del realismo artefactual, no estaría de acuerdo con estas propuestas deflacionarias.

c) El problema del formalismo es que la no-contradicción es condición necesaria, pero no suficiente de la existencia. Esto fue advertido claramente por Frege al sostener que la existencia es un predicado de segundo orden, esto es, un concepto. La existencia es una propiedad de un concepto. Un objeto, en cambio, es lo que cae bajo un concepto. Se sigue de lo anterior que un objeto podría ser no-contradictorio (como la montaña de oro de Meinong), pero aun así inexistente desde el punto de vista ontológico e imposible lógicamente, como las monedas perdidas y la rueda herrumbrada de Tlön; el disco de Odín en El disco; la Biblioteca de Babel en el cuento homónimo o el pensamiento de los Yahoos en El informe de Brodie.

d) Las nociones de inconsistencia y contradicción no poseen contornos claros y delimitados en todos los casos de instanciación. En Tigres azules las piedras son consistentes e inconsistentes al mismo tiempo: son consistentes en cuanto a sus propiedades físico-químicas y geométricas, pero son inconsistentes al contradecir nuestras leyes físicas y aritméticas. Los discos azules corresponderían a un tipo de mundo imposible, pero en un sentido restringido o limitado, esto es, sólo en cuanto se rigen por leyes físicas y reglas aritméticas diferentes a las de nuestro mundo real.

La reflexiones anteriores parecen sugerir la idea de que cuando hablamos de «mundos imposibles», lo que queremos significar es la idea de un mundo lógicamente imposible, en el sentido que las leyes de la lógica clásica no se cumplen en ese mundo (principalmente el principio de no-contradicción). Pero ¿qué queremos decir exactamente con mundos lógicamente imposibles? Hay aquí, a lo menos, cuatro respuestas:

(i) La primera tiene lugar en el contexto de una conversación ordinaria, cuando imaginamos cómo es que las cosas podrían haber sido de otro modo (por ejemplo, cómo sería el mundo si Alemania hubiese ganado la segunda guerra mundial).

(ii) Otra alternativa consiste en sostener que los mundos lógicamente imposibles son aquellos en los que fallan las leyes de la lógica. Así, dada 
una cierta lógica $\mathrm{L}$, un mundo imposible con respecto a las leyes de esa lógica $\mathrm{L}$ es uno en el cual esas leyes no pueden sostenerse (Priest, 1992).

(iii) Una tercera opción es afirmar que un mundo imposible es sólo aquel en el que las leyes de la lógica clásica no se cumplen (Priest, 1997).

(iv) Por último, los mundos imposibles pueden ser definidos como aquellos en los que se da cuenta de contradicciones lógicas explícitas, vulnerándose el principio de no contradicción. Un mundo imposible en este sentido lo será también en (iii), pero no necesariamente a la inversa.

\section{Dialeteísmo y lógica paraconsistente}

El dialeteísmo es la doctrina que afirma que hay contradicciones verdaderas ${ }^{7}$. Según sus defensores el principio de no-contradicción algunas veces falla, entre otras razones, porque el mundo sería inconsistente.

Por su parte, un sistema de lógica paraconsistente es aquél que tolera la contradicción. La idea es similar a la propuesta dialeteísta, pero no debemos confundir las cosas: un lógico paraconsistente no necesita suscribir el dialeteísmo; en cambio, el dialeteísta precisa de un sistema de lógica paraconsistente si quiere ser tomado en serio. La diferencia radica en que el dialeteísmo es una doctrina filosófica, mientras que la lógica paraconsistente es un sistema de lógica formal, donde lo esencial es la invalidación del llamado principio de explosión ${ }^{8}$ de la lógica clásica:

$$
\mathrm{A} \wedge \neg \mathrm{A} \vdash \mathrm{B}
$$

Desde Aristóteles, pasando por la demostración de Duns Escoto, la lógica clásica trivializa la contradicción, pues entiende que si ella tiene lugar, "B" puede ser reemplazado por cualquier proposición bien lograda de nuestro lenguaje, lo que conduce a un resultado absurdo. La virtud de los sistemas de lógica paraconsistente es que admiten la contradicción, pero no por ello originan sistemas triviales o delicuescentes, lo cual supone distinguir nítidamente inconsistencia de trivialidad (Palau y Durán 2009: 358)9.

\footnotetext{
${ }^{7}$ No debe confundirse el dialeteísmo con el trivialismo, que es la doctrina que afirma que todas las contradicciones son verdaderas. El dialeteísta defiende que sólo algunas contradicciones son verdaderas.

${ }^{8}$ Ex contradictione (sequitur) quodlibet, usualmente abreviado como ECQ.

${ }^{9}$ En rigor, la única condición necesaria y suficiente para el desarrollo de un sistema de lógica paraconsis-
} 


\subsection{Del gato de Schrödinger a los tigres de Borges}

En Tigres azules Borges retoma el problema filosófico del cambio y el movimiento, al cual se había abocado en La perpetua carrera de Aquiles y la tortuga (Borges, 2011b: 525-29) y en Avatares de la tortuga (Borges, 2011c: 536-41), pero lo presenta de un modo diferente y original. En lugar de las paradojas de Zenón y el problema del infinito, en Tigres azules la preocupación de Borges se centra no en la multiplicación o división infinita de las piedras, sino en que éstas -al multiplicarse, dividirse, restarse o sumarse- no parecen obedecer ningún patrón ni orden racional, al punto que la Aritmética y el cálculo son imposibles. Peor aún, las piedras son capaces de engendrar, pues "tres y uno pueden ser dos o pueden ser catorce". Surge aquí una pregunta ontológica que posee consecuencias semánticas: en el instante que las piedras azules "cambian", ¿los objetos son o no las mismas piedras?

Supongamos que $t$ representa el enunciado «ahora estoy saliendo de la habitación». La pregunta es: ¿estoy afuera o adentro de la habitación en el instante $t$ ? De acuerdo con Priest hay cuatro alternativas:

1. Estoy adentro.

2. Estoy afuera.

3. Estoy adentro y afuera.

4. No estoy ni adentro ni afuera.

Un defensor de la lógica clásica se vería forzado a elegir entre (1) o (2). El dialeteísta, en cambio, tiene las opciones (3) y (4). Priest argumenta que como no existe una forma de determinar el valor de verdad de (1) y (2) sin recibir una acusación de arbitrariedad, pues no sabemos factual o semánticamente si las proposiciones son verdaderas o falsas, deberíamos abandonar la semántica bivalente de la lógica clásica y optar por los enunciados (3) o (4). Ahora bien, debido a que no puede haber vacíos de verdad (gaps), la sugerencia de Priest es adoptar (3), esto es, el planteo del dialeteísmo. Su principal argumento para descartar (4) es el principio de exhaustividad, conforme al cual «si A no es verdadero, entonces $\neg$ A es verdadero».

tente es el rechazo del principio de explosión (ECQ). El abandono del principio de no contradicción no es una condición necesaria para adscribir a la paraconsistencia. De hecho, el sistema LP de Graham Priest no rechaza el principio de no-contradicción: sólo invalida ECQ tratándose de algunas contradicciones que considera simultáneamente verdaderas. 
En el instante en que las piedras cambian, ¿los objetos son o no las mismas piedras? Tenemos las mismas cuatro respuestas :

5. En el instante de cambio, los objetos son las mismas piedras.

6. En el instante de cambio, los objetos no son las mismas piedras.

7. En el instante de cambio, los objetos son y no son las mismas piedras.

8. En el instante de cambio, los objetos ni son ni no son las mismas piedras.

Y la misma sugerencia: el planteo dialeteísta de (7) en lugar del indiferentismo de (8).

Como observa Tajer (2014: 97) el argumento de Priest presenta varios problemas, que no puedo desarrollar aquí. Su propuesta es fuertemente contraintuitiva, ya que supone optar por un estado de cosas en que la proposición es verdadera y falsa simultáneamente. A esta última situación Priest la llama gluts ("atasco"), mientras que al estado de cosas representado en los enunciados (4) y (8) lo denomina gap. Sin embargo, no puede soslayarse la notable similitud del argumento de Priest con el famoso experimento mental que Edwin Schrodinger inventó en 1935, para exponer una de las interpretaciones más contraintuitivas de la mecánica cuántica. Y es particularmente notable que el experimento sea conocido también como la "paradoja" de Schrödinger, y que dos felinos - un gato y unos tigres- permitan explicar y, quizás superar, las contradicciones de dos porciones de la realidad tan disímiles como la física teórica y la ficción literaria.

\subsection{La semántica de la lógica de las paradojas LP}

En lógica clásica contamos con dos valores de verdad: verdadero (1) y falso (0). En su libro An introduction to Non-Classical Logic, Priest introduce un tercer valor de verdad "i”". Luego considera la semántica de Kleene (K3) y el sistema lógico de Lukasiewicz (L3) y clasifica en dos grupos a las lógicas trivalentes:

1. Las que interpretan el valor de verdad "i" como un gap ("ni verdadero ni falso"): K3 y L3.

2. Las lógicas como LP, que interpretan "i”" como un glut ("verdadero y falso").

LP tiene casi la misma estructura que $\mathrm{K} 3:[\mathrm{V}, \mathrm{D},\{\mathrm{fc} ; \mathrm{c} \in \mathrm{C}\}]$ donde, 
C corresponde a las conectivas de la lógica proposicional $\{\wedge, \vee, \neg, \supset\}$

$\mathbf{V}$ es el conjunto de los valores de verdad $\{0,1, i\}$

D es el conjunto de los valores designados $\{1, \mathrm{i}\}$; éstos son los valores que se preservan en una inferencia válida.

Para cada conectiva $c$, $f c$ es la función veritativa que denota. Por ejemplo: $\mathrm{f} \neg$ es una función de un solo lugar tal que $\mathrm{f} \neg(0)=1, \mathrm{f} \neg(1)=0 \mathrm{y} \mathrm{f} \neg$ (i) $=\mathrm{i}$

Una de las diferencias entre la semántica de Kleene y la lógica de las paradojas LP radica en el conjunto de valores designados. En K3 el conjunto de valores designados $D$ es $\{1\}$; en LP será $\{1, \mathrm{i}\}$. Por eso, en K3 la ley del tercero excluido será inválida, pero no lo será en LP. Otra característica de LP es que el modus ponens no es una inferencia válida.

\section{Conclusiones}

En la lectura de Tigres azules que he propuesto en este trabajo, tanto la lógica como los problemas ontológicos y semánticos constituyen la esencia de su línea argumental. Sin embargo, lo singular del cuento no reside sólo en esa circunstancia, ya que Borges mostró siempre un especial interés por la lógica, el lenguaje y la metafísica. La particularidad de Tigres azules radica en la forma en que Borges construye las paradojas, que no son autorreferenciales, sino variantes de la paradoja de Moore y de las paradojas de implicación material. No se trata, entonces, de que el cuento tenga una "estructura paradójica" originada en la dicotomía orden-caos o la oposición entre lo racional y lo irracional. Una interpretación de este tipo subrayaría la confrontación entre la lógica clásica (con sus principios de identidad, tercero excluido y no-contradicción) y el mito ficcional (representado por las piedras que destruyen la ciencia y hacen imposible el cálculo y la Aritmética). Desde mi punto de vista, la naturaleza paradojal del cuento deriva de que, en la segunda parte, Borges articula paradojas que no pueden resolverse mediante la lógica clásica y sus extensiones, siendo necesario acudir a lógicas no-clásicas. Lo esencial en mi lectura es que en Tigres azules nunca abandonamos los dominios de la lógica.

Desde el punto de vista ontológico, las piedras azules son objetos imposibles debido a su naturaleza autocontradictoria. En la dimensión semántica, estos objetos engendrarían mundos imposibles en un sentido restringido, esto es, mundos donde las leyes de la lógica clásica no se cumplen (no sólo el principio de no contradicción, sino también el principio de identidad: vid. supra 
4.1). En el caso de la ficción literaria el problema de la verdad y los mundos posibles es incluso más complejo, entre otras razones, porque el "imposible lógico" no necesariamente equivale al "imposible ficcional". Por otro lado, como observa D. Lewis, en el caso de la ficción existe una diferencia conceptual entre los mundos imposibles por trama y por argumento, y los mundos inconsistentes. La verdad en estos mundos respondería a un criterio vacuo de verdad, esto es, algo que no es ni verdadero ni falso, sino sencillamente indefinido (en la terminología de Priest correspondería a gaps).

Russell afirmó que la lógica no puede admitir un unicornio más de lo que lo admite la zoología. Tal parece que la lógica no sólo ha de admitir unicornios en la luna, sino que también ha de admitir que en la luna seis o siete unicornios pueden ser tres.

\section{Referencias bibliográficas}

Borges, J. L. (1996a). “Tigres azules”, en Obras completas, Tomo 3. Buenos Aires: Emecé Editores, pp. 381-388.

Borges, J. L. (1996b). "El libro de arena", en Obras completas, Tomo 3. Buenos Aires: Emecé Editores, pp. 11-73.

Borges, J. L. (1996c). "Kafka y sus precursores", en Obras completas, Tomo 2. Buenos Aires: Emecé Editores, pp. 88-90.

Borges, J. L. (1996d). "El idioma analítico de John Wilkins", en Obras completas, Tomo 2. Buenos Aires: Emecé Editores, pp. 84-87.

Borges, J. L. (2011a). “Tlön, Uqbar, Orbis Tertius”, en Obras completas, Tomo 1. Buenos Aires: Editorial Sudamericana, pp. 724-736.

Borges, J. L. (2011b). "La perpetua carrera de Aquiles y la tortuga", en Obras completas, Tomo 1. Buenos Aires: Editorial Sudamericana, pp. 525529.

Borges, J. L. (2011c). "Avatares de la tortuga”, en Obras completas, Tomo 1. Buenos Aires: Editorial Sudamericana, pp. 536-541.

Bulacio, C. (2004). Como el rojo Adán del paraíso. Ensayo de antropología filosófica. Buenos Aires: Libros del Zorzal.

De Toro, A. (1999). “¿Paradoja o rizoma? 'Transversalidad' y 'escriptibilidad' en el discurso borgeano", en El siglo de Borges: homenaje a 
Jorge Luis Borges en su centenario, Alfonso de Toro (coord..), Vol. 1: Retrospectiva, presente, futuro. Madrid: Iberoamericana/Vervuert, pp. 173-208.

Díaz-Estévez E. (1972). "La noción de paradoja y la autorreferencialidad". Anuario Filosófico, 5, pp. 57-96.

Frege, G. (1972). Conceptografía. Los fundamentos de la Aritmética. Otros estudios filosóficos. México: Instituto de Investigaciones Filosóficas.

Hintikka, J. (1962). Saber y Creer. Madrid: Editorial Tecnos, 1979.

Kcenich, Stephen \& Luna-Escudero-Alié, María-Elvira (2014). "Variable aleatoria discreta o 'la aberración de las matemáticas' en 'Tigres azules' y los límites del lenguaje en 'El espejo y la máscara' de Jorge Luis Borges", en Proyecto Patrimonio. Consulta el 19 de febrero de 2016: http://letras.s5.com/jlbo091014.html

Lewis, D. (1986). On the Plurality of Worlds. Oxford: Basil Blackwell.

Millán-Puelles, A. (1990). Obras completas. Vol. VIII, Teoría del objeto puro. Madrid: Ediciones Rialp.

Palau, Gladys \& Durán, Cecilia (2009). "El Significado de la Negación Paraconsistente", Principia, 13 (3), 357.

Pradilla, M. (2015). "Paradojas lógicas y semánticas", en Revista Ingeniería, Matemática y Ciencias de la Información, Vol. 2 Núm. 3.

Priest, G. (1992). “¿What is a Non-Normal World?”, Logique et Analyse, 35: 291-302.

Priest, G. (1997). “Editor's Introduction”, Notre Dame Journal of Formal Logic, 38: 481-7.

Priest, G. (2001). An introduction to Non-Classical Logic. Cambridge: Cambridge University Press.

Sarlo, B. (2007). Borges, un escritor en las orillas. Madrid: Siglo XXI Editores.

Shoemaker, S. (1995). "Moore's Paradox and Self-Knowledge", en The First-person Perspective and Other Essays. Cambridge: Cambridge University Press, pp. 74-93.

Stepanians, M. (2007). Gotllob Frege: Una introducción. Cuadernos de Lógica, Epistemología y Lenguaje, Vol. 1. London: College Publications. 
Tajer, D. (2014). “Alcances y problemas del dialeteísmo”, en Tópicos, Santa Fe, n. 28, p. 89-111.

Thiher, A. (2005). Fiction Refracts Science: Modernist Writers from Proust to Borges. Columbia, Misuri: University of Missouri Press. 
